\title{
Augmenting Cough via Home Ventilators in Subjects With Neuromuscular Disease: Simple, Effective, and Clever
}

Providing noninvasive ventilation in subjects with weak respiratory muscles underlies the availability of effective noninvasive airway clearance techniques during respiratory tract infections. The study by Del Amo Castrillo et $\mathrm{al}^{1}$ in this issue of the Journal confirms the relevance of the principle that associates noninvasive techniques of home mechanical ventilation and cough augmentation in subjects affected by neuromuscular disorders. This strategy is not new; $\mathrm{Bach}^{2}$ and $\mathrm{Bach}^{\mathrm{at}} \mathrm{al}^{3}$ were pioneers in support of the idea that the 2 techniques belong together.

Available techniques of airway clearance offer variable cost-effectiveness ratios. I am convinced that the investment in expensive techniques must be justified clinically. In a bid to reduce the number and cost of respiratory devices at home, a group of experts found that devices for cough assistance are not essential to augment cough in every subject. ${ }^{4} \mathrm{~A}$ recent expert meeting report on airway clearance techniques in neuromuscular disorders highlighted that the unequivocal need for such devices should be restricted to subjects who are weaker.

Cough efficiency in adult subjects depends on the capacity to produce peak expiratory flows during cough, which are $>180 \mathrm{~L} / \mathrm{min}$ during respiratory tract infections. However, this limit of effectiveness is based on expertise rather than on evidence. ${ }^{5}$ Fortunately, increasing the inspiratory capacity alone, before coughing, is a smart and inexpensive way to augment cough in subjects with muscular disorders. This can be achieved by either a manual resuscitator or a volume ventilator. In a variety of ways, the study conducted by Del Amo Castrillo et $\mathrm{al}^{1}$ contributes to our knowledge regarding the optimal way to increase the inspiratory capacity and, therefore, to improve the coughing capacity of subjects with neuromuscular disorders.

Two strategies that aim at augmenting the inspiratory capacity by using a volume ventilator were compared:

\footnotetext{
The author has disclosed no conflicts of interest.
}

Correspondence: Michel Toussaint PT PhD, Rehabilitation Hospital Inkendaal, Inkendaalstraat, 1, 1602 Vlezenbeek, Belgium. E-mail: michel.toussaint@inkendaal.be.

DOI: $10.4187 /$ respcare. 06942 breath-stacking versus volumetric cough mode. Breathstacking consisted of the inhalation of multiple and consecutive insufflations without exhaling. In this strategy,

See the Original Study on Page 255

the control of glottal closure was essential. During volumetric cough mode, the subjects intermittently inhaled a single volume of air that was greater than the baseline tidal volume set on the ventilator. Glottal competency, therefore, was not essential. Cough effectiveness that resulted from the 2 strategies was measured by the cough peak flows and rated by the subjects via a score of tolerance, including the comfort and effectiveness of coughing.

In general, the authors concluded that neither strategy was superior and that both methods deserve to be evaluated to identify the best method for each subject. Data analysis indicated that all the enrolled subjects were able to improve their cough capacity with at least one technique. ${ }^{1}$ Even if the interpretation of the data requires a word of caution due to the small number of participants included in the study and the potential air leaks during the trials, the results indicated that the volumetric cough mode was the first choice to augment cough under the following conditions:

- When subjects do not improve their spontaneous cough peak flow with breath-stacking

- When the spontaneous cough peak flow is $<180 \mathrm{~L} / \mathrm{min}$

- When bulbar function is impaired

This means that, when subjects with neuromuscular disorder are not able to breath-stack or when they have an ineffective cough due to low cough peak flow or bulbar impairment, the single-cycle insufflation via a volume ventilator is a priori the best strategy to improve cough. This is valuable information for clinicians.

During the volumetric cough mode trials, $100 \%$ of subjects were able to inhale a greater volume than the tidal volume set on the ventilator. In addition, $80 \%$ of the subjects inhaled at least twice this tidal volume. ${ }^{1}$ In the breathstacking mode, $85 \%$ of the individuals were able to stack multiple breaths and $25 \%$ were able to stack $\geq 3$ cycles from the ventilator. The subjects performed a median of 


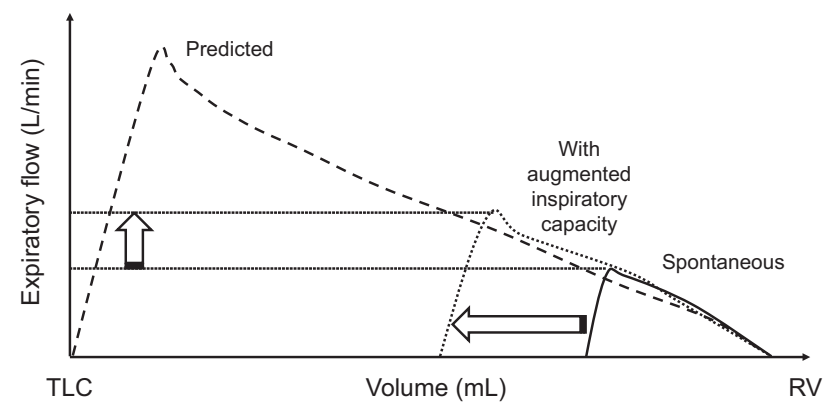

Fig. 1. Increasing inspiratory volume increases peak expiratory flow during cough. TLC $=$ total lung capacity, $\mathrm{RV}=$ residual volume.

2 breaths stacked, which is in line with a previous study that reported a mean of 1.8 and 2.6 breaths before coughing by using either a resuscitator bag or a ventilator, respectively. ${ }^{6}$ This is relevant information for clinicians to tailor the appropriate number of stacked inspiratory cycles before coughing.

I found it interesting that the authors asked subjects to score their breathing comfort and effectiveness with the 2 strategies. Despite volumes and flows often being lower after multiple insufflations compared with a single insufflation, the subjects did not score the latter more favorably. It was originally hypothesized that volumetric cough mode might be better tolerated than breath-stacking. However, this hypothesis was not confirmed. Higher insufflations and flows did not result in higher subject satisfaction, which indicates that the ideal strategy must be discussed with subjects to find a compromise between objective and subjective findings. Again, our study supports the need for individualization of respiratory techniques. ${ }^{6}$ Relevant trends can be available for groups of subjects, but there is still a need to tailor the technique to every individual.

The present study ${ }^{1}$ also confirms several important findings of previous reports. ${ }^{4,5}$ First, pressure-cycled ventilators are not suitable for breath-stacking. Multiple stacked breaths are impossible to achieve when inspiratory cycles are pressure limited. By definition, stacking successive insufflations in the lungs implies the possibility to reach higher pressures than those set on the device. Volumecycled breaths offer this possibility. This is the reason why the authors selected the volume mode of ventilation to augment cough.

Second, the findings of Del Amo Castrillo et al ${ }^{1}$ confirm the positive relationship between the inspiratory volume and the peak expiratory flow during cough. This relationship is illustrated in Figure 1. Forced expiratory flow that results from an augmented insufflation was higher than the flow obtained from a spontaneous breath. The higher inspiratory volume before coughing yields the highest peak flow during cough maneuvers.
Caution regarding the measurement of the inspiratory capacity is needed. First, the delivered tidal volume does not always compare with the volume set on the device, even during volume control mode. ${ }^{7}$ For this reason, it is unclear whether subjects received the tidal volume set on the ventilator. Second, significant leakage via the mouth is highly likely to occur after disconnection from the ventilator. For that reason, the calculated delivered inspiratory capacity may not reflect the actual inspiratory capacity that the subjects were able to inhale. I agree with the authors that the inspiratory capacity may have been overestimated in some cases. I wonder why the authors did not report the expiratory volume during cough because they measured the expiratory flow. Reporting actual exhaled volumes would give valuable information regarding the percentage of leaks by comparing the actual exhaled and the calculated (delivered) inspiratory capacity.

Finally, this study is a reminder of the need for strong indicators of effective cough. ${ }^{1}$ Expiratory cough flows $>270 \mathrm{~L} / \mathrm{min}$ were achieved by only $15 \%$ of the subjects after breath-stacking, whereas $80 \%$ judged their cough as effective (score $\geq 5 / 10$ ). ${ }^{1}$ This highlights that the limit of effective cough generally admitted at a cough peak flow $>270 \mathrm{~L} / \mathrm{min}$ is probably overestimated. Studies that aim at differentiating those subjects able to cough from those subjects unable to cough are still necessary. In 2006, the French Authorities for Health suggested that the $270 \mathrm{~L} / \mathrm{min}$ threshold value for effective cough should be reduced to an estimated $180 \mathrm{~L} / \mathrm{min} .{ }^{8} \mathrm{In}$ summary, augmenting the insufflation capacity via a volume ventilator is a simple, effective, and clever way to augment cough in subjects with neuromuscular disorders.

Michel Toussaint Centre for Home Mechanical Ventilation and Neuromuscular Disorders Department of Rehabilitation, Rehabilitation Hospital Inkendaal Vlezenbeek, Belgium

\section{REFERENCES}

1. Del Amo Castrillo L, Lacombe M, Boré A, Vaugier I, Falaize L, Orlikowski D, et al. Comparison of two cough-augmentation techniques delivered by home ventilator in subjects with neuromuscular disease. Respir Care 2019;64(3):255-261.

2. Bach JR. A historical perspective on the use of noninvasive ventilatory support alternatives. Respir Care Clin N Am 1996;2(2):161-181.

3. Bach JR, Barrow SE, Goncalves M. A historical perspective on expiratory muscle aids and their impact on home care. Am J Phys Med Rehabil 2013;92(10):930-941.

4. Toussaint M, Chatwin M, Gonzales J, Berlowitz DJ, ENMC Respiratory Therapy Consortium. 228th ENMC International Workshop: 


\section{EDITORIALS}

Airway clearance techniques in neuromuscular disorders Naarden, The Netherlands, 3-5 March, 2017. Neuromusc Disord 2018;28(3): 289-298.

5. Chatwin M, Toussaint M, Gonçalves MR, Sheers N, Mellies U, Gonzales-Bermejo J, et al. Airway clearance techniques in neuromuscular disorders: a state of the art review. Respir Med 2018;136(Can't get issue from record): $98-110$.

6. Toussaint M, Pernet K, Steens M, Haan J, Sheers N. Cough augmentation in subjects with Duchenne muscular dystrophy: comparison of air stacking via a resuscitator bag versus mechanical ventilation. Respir Care 2016;61(1):61-67.

7. Ogna A, Prigent H, Falaize L, Leroux K, Santos D, Vaugier I, et al. Accuracy of tidal volume delivered by home mechanical ventilation during mouthpiece ventilation: a bench evaluation. Chron Respir Dis 2016; 13(4):353-360.

8. Leger P, Paulus J. [Recommendations of HAS: Practical issues in home non-invasive ventilation in patients with neuromuscular disease]. Rev Mal Respir 2006;23(4 Suppl):13S141-13S143. 